\title{
TOPOLOGIES WITH THE STONE-WEIERSTRASS PROPERTY $\left({ }^{1}\right)$
}

BY

PAUL R. MEYER

The two main results in this paper are analogues of the Stone-Weierstrass theorem for real-valued functions, obtained by using different function space topologies. The first (Theorem 2.3) is a Stone-Weierstrass theorem for unbounded functions. The second (Theorem 3.6) is a theorem for bounded functions; it is stronger than the usual theorem because the topology is larger than the uniform topology. In fact it is the strongest possible theorem in a sense made precise in Theorem 3.5. However, the usefulness of the result is, in both cases, limited by the fact that the topologies used are far from being as well behaved as the uniform topology. Some specific defects of these topologies are discussed in Proposition 2.4, Proposition 3.9, and Example 3.10. (The topologies are defined in §1.)

The formulation of the Stone-Weierstrass theorem on which the analogues in the present paper are based is the following: If $X$ is a completely regular topological space, then $X$ is compact iff every strongly separating subalgebra of $C^{*}(X)$ is $u$-dense, where $u$ denotes the uniform topology. In [5] Lorch has studied a more general class of function algebras than those of the form $C^{*}(X)$. All of the results for bounded functions in this paper are formulated in this more general setting (see §1).

Terminology and notation. Compact and completely regular topological spaces are assumed to be Hausdorff. The sequence $f_{1}, f_{2}, \ldots$ is denoted by $\left(f_{n}\right)$. In general, when no indexing set is specified, it is understood to be the set $N$ of positive integers. When lattice notation is used for topologies on a set, it is understood that the lattice in question is the lattice of all topologies on the set.

1. Some function space topologies. Let $R^{X}$ denote the set of all real-valued functions on $X$ and $\left(R^{X}\right)^{*}$ denote the set of all bounded such functions. Following Lorch [5], we let $B$ be a subalgebra of $\left(R^{X}\right)^{*}$ which contains the constants, separates points, and is complete in the sup norm. Note that $B$ is a sublattice. The topology on $X$ is always understood to be the weak $B$ topology (called the $\beta$-topology by Lorch). For the special case in which $B$ includes all of the bounded $\beta$-continuous functions, we follow the usual notation and write $C^{*}(X)$ or $C^{*}$ instead of $B$. This

Presented to the Society, April 20, 1964; received by the editors February 15, 1966.

(1) This paper contains the second portion of the author's doctoral dissertation, which was prepared at Columbia University under the direction of Professor E. R. Lorch. The author is pleased to acknowledge his debt to Professor Lorch. Much of the work was done during the tenure of a National Science Foundation Fellowship. These results were announced in [6]. 
paper can be read independently of Lorch's paper if one restricts consideration to this special case. In dealing with unbounded functions we consider only the one case: $C(X)$ or $C$ denotes the set of all continuous functions on the completely regular topological space $(X, \beta)$.

For use in $\S 2$, we define a class of topologies as follows: If $S$ is a set of functions on $X$, and $Y$ is a set containing $X$ such that each function in $S$ has a canonical extension to a function on $Y$, then the "topology of pointwise convergence on $Y$ " is meaningful as a topology on $S$. This topology has an nbd base at 0 consisting of sets of the following form:

$$
U(0, A, \varepsilon)=\{f \in S:|f(x)|<\varepsilon \text { for each } x \text { in } A\}
$$

where $A$ is any finite subset of $Y$ and $\varepsilon>0$. Let $p$ denote the usual topology on $R^{X}$ of pointwise convergence on $X$. Let $p^{\prime}$ (respectively $p^{\backslash}, p^{\wedge}$ ) denote the topólogy on $B$ of pointwise convergence on $X^{\prime}$ (respectively $X^{\backslash}, X^{\wedge}$ ). (For the case $B=C^{*}(X)$ we have, in the notation of [3], $X^{\prime}=v X, X^{\backslash}=X \cup(\beta X-v X)$ and $X^{\wedge}=\beta X$.) The topology $p^{\prime}$ is also meaningful as a topology on $C(X)$, because the functions in $C(X)$ can be extended to $v X$, the realcompactification of $X$.

The topology $p^{\wedge}$ has been used by Brace in a different context [1].

The following proposition is proved in [8].

Proposition 1.1. Every strongly separating subalgebra of $R^{X}$ is p-dense in $R^{X}$.

For use in $\S 3$, let $u_{m}$ denote the largest topology on $\left(R^{X}\right)^{*}$ in which uniformly convergent monotone sequences are topologically convergent. (Let $u$ denote the usual uniform topology, which can be described as the largest topology in which uniformly convergent sequences are topologically convergent. It will be apparent later (see Proposition 3.9) that $u_{m}$ is always strictly larger than $u$.) The $u_{m}$ topology can be described by means of the associated Kuratowski closure operator. Let $\mathscr{C}$ denote the class of all uniformly convergent, monotone sequence pairs (i.e., pairs of the form $\left(\left(f_{n}\right), f\right)$, where $\left(f_{n}\right)$ is a sequence in $\left(R^{X}\right)^{*}$ which converges uniformly and monotonely to $f)$. Then $\mathscr{C}$ induces a sequential closure operator on the subsets of $\left(R^{X}\right)^{*}$. This is not a Kuratowski closure operator, because it is not idempotent. However, by iteration of this operator, one obtains the Kuratowski closure operator of the $u_{m}$-topology.

The symbol $u_{m}$ will also be used to denote the relative topology on $B$. (This convention is used for the other function space topologies as well.) Since $B$ is $u_{m}$-closed, the above description of the $u_{m}$-closure operator remains valid for this relative topology if we replace $\mathscr{C}$ by its trace on $B$.

This gives rise to an extension of the classical notion of "Baire order." If $S$ is any subset of $B$ and $f$ is in $u_{m}$-cl $S$ (in $B$ ), then there exists a smallest ordinal $\eta$ such that $f$ is in the $\eta$-th iterate of the sequential closure of $S$ in $B$. Then $f$ is said to have Baire order $\eta$ (write ord $f=\eta$ ) with respect to $S$ and $\mathscr{C}$. Thus a function has a Baire order with respect to each set of which it is a $u_{m}$-limit point. 
The introduction of Baire order here enables us to establish properties of $u_{m}$ by transfinite induction. In [8] a slightly different topology is used for applications more closely related to the original notion of Baire order. These statements about $u_{m}$ are easily verified; however a fuller discussion in several more general contexts is given in [7]. The basic properties of the largest topology in which a specified class of sequences converge are well set forth by R. M. Dudley in [2].

2. A Stone-Weierstrass theorem for unbounded functions. Before considering the unbounded case, we begin with some related results for bounded functions. Following [5] and recalling that each function in $B$ has a canonical extension to a function on $X^{\prime}$, we let $B^{\prime}$ denote the set of all such extended functions; $B^{\prime}$ and $B^{\wedge}$ are defined similarly.

Proposition 2.1. The various pointwise topologies on $B$ are related as follows: $p^{\wedge} \supset p^{\prime} \supset p$ and $p^{\wedge} \supset p^{\backslash} \supset p$. Furthermore, $p^{\prime} \cap p^{\prime}=p$ and $p^{\prime} \vee p^{\prime}=p^{\wedge}$ (i.e., $p^{\prime} \cup p^{\backslash}$ is a subbase of $\left.p^{\wedge}\right) . X$ is realcompact iff $p^{\prime}=p$ on $B ; X$ is pseudocompact iff $p^{\backslash}=p$ on $B$; $X$ is compact iff $p^{\wedge}=p$ on $B$. The extension mapping is a homeomorphism from $\left(B, p^{\prime}\right)$ to $\left(B^{\prime}, p\right)$; there is a similar homeomorphism in each of the other two cases.

Proof. We shall prove the assertion: If $X$ is not realcompact, then $p^{\prime} \neq p$ on $B$. The other proofs are either similar to this one or else follow directly from the definition of the nbd base. Since $X$ is not realcompact, we can choose $y$ in $X^{\prime}-X$. The set $U(0,\{y\}, 1 / 2)$ is a $p^{\prime}$-nbd 0 . To show that $U(0,\{y\}, 1 / 2)$ does not contain any basic $p$-nbd 0 , let $U(0, A, \varepsilon)$ be any such. Say $A=\left\{x_{1}, \ldots, x_{n}\right\} \subset X$. Since $B^{\wedge}$ has the strong separation property, there exist $f_{k}$ in $B$ for $1 \leqq k \leqq n$ such that $f_{k}\left(x_{k}\right)=0$ and $f_{k}^{\prime}(y)=1$. Put $f=f_{1} f_{2} \cdots f_{n}$. Then $f$ is in $U(0, A, \varepsilon)$ and not in $U(0,\{y\}, 1 / 2)$, which proves that $p \neq p^{\prime}$.

Proposition 2.2. (a) $X$ is realcompact iff every strongly separating subalgebra of $B$ is $p^{\prime}$-dense in $B$.

(b) $X$ is pseudocompact iff every strongly separating subalgebra of $B$ is $p^{\backslash}$-dense in $B$.

(c) $X$ is compact iff every strongly separating subalgebra of $B$ is $p^{\wedge}$-dense in $B$.

Before giving the easy proof, let us note that, for the case $B=C^{*}(X)$, (c) asserts that the Stone-Weierstrass theorem is valid for the $p^{\wedge}$-topology. Hence there is more than one "Stone-Weierstrass topology" for $B$. This aspect will be considered in more detail in $\$ 3$.

Proof. We prove (a); the proofs of (b) and (c) are analogous. If $X$ is realcompact then $p^{\prime}=p$, and the result follows from Proposition 1.1. Conversely, if $X$ is not realcompact, then we can choose $y$ in $X^{\prime}-X$. Let $S=\left\{f \in B: f^{\prime}(y)=0\right\}$. Clearly $S$ is a proper subalgebra of $B$ with the strong separation property (for $X$ ). To complete the proof we show that $S$ is $p^{\prime}$-closed. Let $f \in B$ and assume that every $p^{\prime}$-nbd $f$ meets $S$; we show that $f$ is then in $S$. By assumption, $U(f,\{y\}, 1 / n)$ meets $S$ for all 
$n$, and for each $n$ there exists $g_{n}$ in $S$ such that $\left|f^{\prime}(y)-g_{n}^{\prime}(y)\right|<1 / n$. But $g_{n}^{\prime}(y)=0$ and hence $\left|f^{\prime}(y)\right|<1 / n$ for each $n$. Therefore $f^{\prime}(y)=0$ and $f$ is in $S$.

The situation for unbounded functions is not essentially different in the case of the $p^{\prime}$-topology. It is well defined on $C(X)$ and the properties developed in Proposition 2.1 remain valid when $B$ is replaced by $C$. (On the other hand, the topologies $p^{\curlywedge}$ and $p^{\wedge}$ have no meaning in the unbounded case.) By a proof essentially the same as that given above, we obtain

THEOREM 2.3. For a completely regular topological space $X, X$ is realcompact iff every strongly separating subalgebra of $C(X)$ is $p^{\prime}$-dense in $C(X)$.

We now consider the $p^{\prime}$-topology with respect to some of the desirable properties possessed by the $u$-topology in the bounded case. Very briefly, we have

Proposition 2.4. Under the $p^{\prime}$-topology, $C(X)$ is a locally convex topological algebra. Maximal ideals in $C(X)$ need not be $p^{\prime}$-closed. If $X$ is not discrete then $C(X)$ is not complete in the p-uniformity.

Proof. The first and last statements are essentially known facts about the $p$ topology. (Recall that $\left(C(X), p^{\prime}\right)$ is homeomorphic to $(C(v X), p)$.) We construct an example of a maximal ideal in $C(N)$ which is not $p^{\prime}$-closed. ( $N$ is the space of positive integers.) Let $f_{n}$ denote the characteristic function of $\{1, \ldots, n\}$. The set $\left\{f_{1}, f_{2}, \ldots\right\}$ generates a proper ideal which is contained in a maximal ideal $M$. But $M$ is not $p^{\prime}$-closed because the sequence $\left(f_{n}\right)$ is $p^{\prime}$-convergent to 1 . (In this case $p^{\prime}=p$.)

3. The largest Stone-Weierstrass topology. It will be convenient to have the following

Definition 3.1. A topology $t$ on $B$ is called an $S W$ topology if the following condition is satisfied: $X$ is compact iff every strongly separating subalgebra of $B$ is $t$-dense in $B$.

In addition to the $u$-topology, the SW topologies include Hewitt's $m$-topology $[3,2 \mathrm{~N}]$ and the $p^{\wedge}$-topology (Proposition 2.2). It is natural to ask whether a given class of topologies contains a maximal or a largest SW topology. (For the distinction between a "largest" element and a "maximal" element see [4, pp. 31-32].)

Proposition 3.2. Let $\mathscr{S}$ denote a class of topologies on $B$ satisfying: (*) If $\left\{t_{i}\right\}_{i \in I}$ is any totally ordered family of topologies in $\mathscr{S}$, then $\bigvee_{i \in I} t_{i}$ is in $S$. Then every SW topology in $\mathscr{S}$ is contained in a topology which is maximal among SW topologies in $\mathscr{S}$.

Proof. The proposition follows from Zorn's lemma with the help of the following general lemma.

LEMMA 3.3. If $B$ is an arbitrary set and $\left\{t_{i}\right\}_{i \in I}$ is any totally ordered family of topologies on $B$, then $t-\mathrm{cl} S=\bigcap_{i \in I} t_{i}-\mathrm{cl} S$, where $S$ is any subset of $B$ and $t=\bigvee_{i \in I} t_{i}$. 
Proof. Since the family is totally ordered, $\bigcup_{i \in I} t_{i}$ is a base for $t$. (Without this restriction it is only a subbase.) The rest of the proof is easy.

One can also define the notion of SW topology for realcompactness or pseudocompactness. Such topologies were shown to exist in Proposition 2.2. The preceding proposition on maximal topologies can be applied to these cases; the same proof is valid.

If $\mathscr{S}$ is the set of all "topological linear space" topologies, then (*) is satisfied. In fact, if $\mathscr{S}$ is any class of topologies on $B$ defined by axioms requiring some form of continuity for the vector space operations, then $(*)$ is satisfied. This follows from another general lemma, the proof of which we omit.

LemMA 3.4. Let $\left\{t_{i}\right\}_{i \in I}$ be a family of topologies on a set $X$. Similarly, let $\left\{u_{i}\right\}$ and $\left\{v_{i}\right\}$ be families of topologies on sets $Y$ and $Z$ respectively. Let $t, u$, and $v$ denote the least upper bounds of the respective families. (These families are not assumed to be totally ordered.) If $F$ is a function on $X \times Y$ to $Z$ which is continuous with respect to $t_{i}, u_{i}$, and $v_{i}$, for each $i$ in $I$, then $F$ is continuous with respect to $t$, $u$, and $v$.

We now show that the topology $u_{m}$ of uniform monotone convergence (defined in $\S 1$ ) is an SW topology and is also the largest topology in a class $\subseteq$ of topologies on $B$. $\subseteq$ can be described roughly as those topologies for which the vector space operations are separately continuous and for which a mild order axiom is satisfied. (The order axiom is a weak form of one used by Namioka [9, Theorem 4.8 (iii)].)

THEOREM 3.5. Let $\subseteq$ be the class of all topologies on $B$ satisfying: (i) The functions $f \rightarrow f+g, f \rightarrow \alpha f$, and $\alpha \rightarrow \alpha e$ are continuous (where $f, g \in B, \alpha \in R$, and $e \in B$ is defined by $e(x)=1$ for all $x$ in $X)$. (ii) If $\left(f_{n}\right)$ and $\left(g_{n}\right)$ are monotone sequences in $B$ such that $f_{n} \geqq g_{n} \geqq f$ and $\left(f_{n}\right)$ converges to $f$, then $\left(g_{n}\right)$ converges to $f$. Then $u_{m}$ is the largest topology in $\mathfrak{S}$.

Proof. It is not difficult to show that $u_{m}$ is in $\mathfrak{S}$. To show that it is the largest element, we assume $t \in \mathcal{S}$ and show that $t \subset u_{m}$. It suffices to show that $\left(\left(f_{n}\right), f\right) \in \mathscr{C}$ implies $\left(f_{n}\right)$ is $t$-convergent to $f$ (since $u_{m}$ is the largest topology with that convergence property). Assume $\left(f_{n}\right)$ is decreasing. Choose $\alpha_{n}$ in $R$ such that $\alpha_{n} \downarrow 0$ and $f+\alpha_{n} e \geqq f_{n} \geqq f$. Then $\left(\alpha_{n} e\right)$ is $t$-convergent to 0 and $\left(f+\alpha_{n} e\right)$ is $t$-convergent to $f$ (by (i)). Therefore $\left(f_{n}\right)$ is $t$-convergent to $f$ (by (ii)).

THEOREM 3.6. The topology $u_{m}$ is an SW topology; i.e., $X$ is compact iff every strongly separating subalgebra of $B$ is $u_{m}$-dense in $B$.

Proof. If $X$ is not compact the proof is trivial, since $u_{m} \supset u$ and $u$ is an SW topology. Assume now that $X$ is compact; it follows that $B$ coincides with $C(X)$. If $S$ is a strongly separating subalgebra of $C$, then $u_{m}$-cl $S$ is a sublattice of $C$ by the following proposition. Hence by the next proposition, $u_{m}$-cl $S$ is $u$-closed. The conclusion now follows from the usual SW theorem. 
Proposition 3.7. If $S$ is any subalgebra of $\left(R^{x}\right)^{*}$, then $u_{m}$-cl $S\left(\right.$ in $\left.\left(R^{x}\right)^{*}\right)$ is a sublattice of $\left(R^{X}\right)^{*}$.

Proof. This proposition has a more general formulation in [7, (3.9)]. For the purpose of this proof, let $S^{-}$denote $u_{m}$-cl $S$ (in $\left(R^{X}\right)^{*}$ ). We begin the proof by making two observations. First, the function $a: x \rightarrow|x|$ on the interval $[-1,1]$ is a uniform monotone limit of polynomials. (This is verified by observing that the usual infinite series expansion converges monotonely.) Second, $S^{-}$is a vector subspace, and for $f$ in $S^{-}$we have ord $f=\operatorname{ord}(f /\|f\|)$, where ord $f$ denotes the Baire order of $f$ as defined in $\$ 1$. (The closure of a vector subspace is a vector subspace whenever $f \rightarrow f+g$ and $f \rightarrow \alpha f$ are continuous. The other part follows by induction on ord $f$.)

To prove the proposition, we must show that $f, g$ in $S^{-}$implies $f \vee g$ is in $S^{-}$. Using the identity $2(f \vee g)=f+g+|f-g|$ and the fact that $S^{-}$is a vector subspace, we can reduce the problem to showing: $f \subset S^{-},\|f\|=1$ implies $|f| \in S^{-}$. We proceed by induction on ord $f$. If ord $f=0$, then $f \in S$. There exist polynomials $p_{n}$ on $[-1,1]$ which converge uniformly and monotonely to $a$. From this it follows that $\left(p_{n}(f),|f|\right) \in \mathscr{C}$. Since $f$ is in $S$ and $S$ is a subalgebra, $p_{n}(f)$ is in $S$. Therefore $|f|$ is in $S^{-}$.

If we now assume that ord $f=\eta>0$, there exists $f_{n} \in S^{-}$such that ord $f_{n}<\eta$ and $\left(\left(f_{n}\right), f\right) \in \mathscr{C}$. The inductive hypothesis asserts that if ord $g<\eta$ and $\|g\|=1$ then $|g| \in S^{-}$. But by our preliminary observation, the restriction $\|g\|=1$ can be removed. Therefore $\left|f_{n}\right| \in S^{-}$. But $\left(\left|f_{n}\right|\right)$ is not a monotone sequence in general, and the argument is not quite finished. Since $\left|f_{n}\right| \in S^{-}, f_{n}^{+}=(1 / 2)\left(\left|f_{n}\right|+f_{n}\right) \in S^{-}$and similarly $f_{n}^{-} \in S^{-}$. From the fact that $\left(\left(f_{n}^{+}\right), f^{+}\right) \in \mathscr{C}$ and $\left(\left(f_{n}^{-}\right), f^{-}\right) \in \mathscr{C}$, it follows that $|f|=f^{+}+f^{-}$is in $S$. This completes the proof of Proposition 3.7.

Proposition 3.8. If $X$ is compact, $S$ is a sublattice of $C(X)$, and $t$ is any topology on $C$ such that $u_{m} \supset t \supset p$, then $t-\mathrm{cl} S=u_{m}-\mathrm{cl} S$ (in $C$ ). (Here there are no continuity assumptions imposed on $t$.)

Proof. Throughout the proof closures are understood to be in $C$. It suffices to show that $p$-cl $S \subset u_{m}$-cl $S$. By the Stone-Weierstrass theorem $p$-cl $S=u$-cl $S$. Assume $f \in u$-cl $S$. Then there exists $g_{n} \in S$ such that $\left(g_{n}\right)$ converges uniformly to $f$. Put $g_{n k}=g_{n} \vee \cdots \vee g_{n+k}$. One can show that there exists $h_{n} \in C$ such that $\left(\left(g_{n k}\right), h_{n}\right) \in \mathscr{C}$. (Since $\left(g_{n k}\right)_{k}$ is uniformly bounded, there exists $h_{n} \in\left(R^{X}\right)^{*}$. The convergence is clearly monotone and can be shown to be uniform; it then follows that $h_{n}$ is continuous.) Then $\left(\left(h_{n}\right), f\right) \in \mathscr{C}$, and $f \in u_{m}$-cl $S$. This completes the proof of Proposition 3.8 and Theorem 3.6.

We now turn to some negative results about the $u_{m}$-topology and a useful geometric description of the $u_{m}$-topology in the case of the plane, $R^{2}$.

Proposition 3.9. Assume that $X$ contains at least two points. Let $S$ be a subalgebra and sublattice of $\left(R^{X}\right)^{*}$ which separates points and contains the constants. Then 
$\left(S, u_{m}\right)$ is not metrizable and not locally convex. Addition in $S$ is not jointly $u_{m}$-continuous; if $f$ changes sign, the function $\alpha \rightarrow \alpha f$ is not continuous on $R$.

Proof. We can choose nonnegative functions $f$ and $g$ in $S$ with nonvoid, disjoint supports [7, (7.2)]. If $T$ is the 2-dimensional subspace of $S$ generated by $f$ and $g$, then $\left(T, u_{m}\right)$ is isomorphic and homeomorphic to $\left(R^{2}, u_{m}\right)$ under the natural mapping. Hence to prove the proposition it suffices to show these negative results for the case of the plane.

EXAMPLE 3.10. In the plane $R^{2}$ the $u_{m}$-open sets can be described geometrically. Let $V_{n}(0)$ be that subset of the open $1 / n$ disk with center at 0 which is obtained by removing all points of the open second and fourth quadrants. For $f \in R^{2}$ let $V_{n}(f)$ be the translation of $V_{n}(0)$ by $f$. Then a subset $W$ of $R^{2}$ is $u_{m}$-open iff $W$ contains some $V_{n}(f)$ for each $f$ in $W$. (Note that $V_{n}(f)$ is not a neighborhood in the usual sense, since it contains no open set containing $f$.) All of the pathological properties mentioned in Proposition 3.9 can be exhibited in this example.

Proof. We begin by showing that the description of the $u_{m}$-open sets is a valid one. This does describe the open sets in some topology; call it $t$. Clearly $t \subset u_{m}$. To show $u_{m} \subset t$, let $W$ be $u_{m}$-open and show that for $f \in W$ there exists $m$ such that $V_{m}(f) \subset W$. Suppose not. Then for each $k$ in $N$ there exists $f_{k} \in V_{k}(f)-W$. We construct a subsequence of $\left(f_{k}\right)$ which is monotonely convergent (and hence $u_{m}$ convergent) to $f$; this contradiction will complete the proof. If $f_{k}=\left(x_{k}, y_{k}\right)$, then there is a subsequence which is monotone in $x$ and a sub-subsequence which is monotone in $x$ and $y$.

We consider now the properties of $\left(R^{2}, u_{m}\right)$. It is clearly not locally convex. (The sets $V_{n}$ are not $u_{m}$-open, but they can be enlarged slightly to remedy this.) To show that the space is not metrizable, it suffices to show that it is not normal. This is done by a counting argument similar to that in $[3,3 \mathrm{~K}]$. The discrete set $D$ $=\{(x, y): y=-x\}$ has cardinality $c$, and every subset of $D$ is a closed $G_{\delta}$. On the other hand, since $\left(R^{2}, u_{m}\right)$ is separable, there are at most $c$ zero sets. Since not every closed $G_{\delta}$ is a zero set, the space is not normal [3, 3D3]. The following examples show that the algebraic operations do not satisfy stronger continuity conditions than those listed in Theorem 3.5. To show that addition is not jointly continuous, let $f_{n}=(1 / n, 0)$ and $g_{n}=(0,-1 / n)$; in this case $\left\{f_{n}+g_{n}\right\}$ is already $u_{m}$-closed (it contains no monotone sequences). Now if $f=(1,-1)$, then $\{(1 / n) f\}$ is $u_{m}$-closed, which shows that $\alpha \rightarrow \alpha f$ is not continuous.

\section{REFERENCES}

1. John W. Brace, The topology of almost uniform convergence, Pacific J. Math. 9 (1959), 643-652

2. R. M. Dudley, On sequential convergence, Trans. Amer. Math. Soc. 112 (1964), 483-507.

3. L. Gillman and M. Jerison, Rings of continuous functions, Van Nostrand, New York, 1960.

4. J. L. Kelley, General topology, Van Nostrand, New York, 1955.

5. E. R. Lorch, Compactification, Baire functions, and Daniell integration, Acta Sci. Math. (Szeged) 24 (1963), 204-218. 
6. P. R. Meyer, Topologies with the Stone-Weierstrass property, Abstract 611-17, Notices Amer. Math. Soc. 11 (1964), 321.

7. - Topologies on spaces of real-valued functions, Ph.D. thesis, Columbia Univ., New York, 1964.

8. - The Baire order problem for compact spaces, Duke Math. J. 33 (1966), 33-40.

9. I. Namioka, Partially ordered linear topological spaces, Mem. Amer. Math. Soc. No. 24, (1957), 50 pp.

Hunter College of the CrTy Universtty of New York, New York, New York 